\title{
LETTER \\ Effectiveness of Speech Mode Adaptation for Improving Dialogue Speech Synthesis
}

\author{
Kazuki KAYA $^{\dagger *}$, Nonmember and Hiroki MORI ${ }^{\dagger a)}$, Member
}

SUMMARY The effectiveness of model adaptation in dialogue speech synthesis is explored. The proposed adaptation method is based on a conversion from a base model learned with a large dataset into a target, dialogue-style speech model. The proposed method is shown to improve the intelligibility of synthesized dialogue speech, while maintaining the speaking style of dialogue.

key words: dialogue, speech synthesis, HMM, transfer learning

\section{Introduction}

Although speech synthesis with astonishing naturalness is now possible with deep neural networks, HMM-based speech synthesis [1], [2] is still advantageous for some niche applications, because it works well for a relatively small corpus, and because it allows flexible customization at various levels thanks to its transparent structure. We have previously studied HMM-based dialogue speech synthesis using a spontaneous dialogue speech corpus as a dataset [3], [4]. Although these studies showed the effectiveness of controlling paralinguistic information in a dimensional representation, they suffered from quality degradation due to insufficient training data.

In this letter, the effectiveness of model adaptation in dialogue speech synthesis is explored. Specifically, the adaptation is achieved by a conversion from a base model learned with a large dataset into a target, dialogue-style speech model.

In this study, we used the Simulated Public Speaking (SPS) subset of the Corpus of Spontaneous Japanese (CSJ) [5] for training the base model, and the Utsunomiya University Spoken Dialogue Database for Paralinguistic Information Studies (UUDB) [6] for adapting the base model into dialogue speech. Although these two datasets differ in speech mode (monologue vs. dialogue), their speaking styles are relatively similar. The main concern of this paper is to what extent the speech mode adaptation overcomes the limitation of the amount of dialogue speech corpora.

\section{Methods}

To examine the effectiveness of speech mode adaptation, the

Manuscript received January 28, 2019.

Manuscript revised May 11, 2019.

Manuscript publicized June 13, 2019.

${ }^{\dagger}$ The authors are with the Graduate School of Engineering, Utsunomiya University, Utsunomiya-shi, 321-8585 Japan.

*Presently, with TOKYO KEIKI INC.

a) E-mail: hiroki@ speech-lab.org (Corresponding author) DOI: 10.1587/transinf.2019EDL8024 following three models were compared:

UUDB Trained with utterances of one female speaker in the UUDB (551 utterances, about 11 minutes).

CSJ Trained with utterances of $\mathbf{5 4}$ female speakers in the SPS subset of CSJ (13266 utterances, about 5 hours 4 minutes).

ADAPT Converted the CSJ model by the MLLR+MAP adaptation, where the 551 utterances of the UUDB were used as the adaptation data.

Speech signals were $16 \mathrm{bit}$ PCM sampled at a rate of $16 \mathrm{kHz}$. The structure of each model was a 5-state left-to-right hidden semi-Markov model (HSMM). The feature vector consisted of 34th-order mel-cepstral coefficients, log F0, and their $\Delta$ and $\Delta \Delta$ features. The mel-cepstrum parameter was obtained by converting from the STRAIGHT spectrum [7]. For the F0 extraction, the pitch tracker in the Snack sound toolkit was used.

For building the CSJ model, the speaker adaptive training (SAT) with shared decision tree [8] was employed in order to guarantee that all leaf nodes are affected by all the speakers in the training set.

As the adaptation method for the ADAPT model, a combination of Maximum Likelihood Linear Regression (MLLR) and Maximum A Posteriori (MAP) estimation [9] was used. As the prior in the MAP estimation, means of Gaussians transformed by MLLR were used. The regression class tree was first created based on a speech-modeindependent model trained with both UUDB and CSJ utterances then the transformations were grouped using the adaptation data, as in typical speaker adaptation [9].

As the result of training, the F0 decision tree of the UUDB model had 352 leaf nodes, and that of the CSJ model had 1951 leaf nodes. The number of nodes of the regression class tree was 111.

\section{Perceptual Evaluation}

\subsection{Experimental Conditions}

It is not sufficient to evaluate dialogue speech synthesis only by naturalness or intelligibility. In this study, we evaluated whether the synthesized speech sounded like dialogue speech, as well as its intelligibility. For each model, 50 utterances were synthesized from the context labels of the UUDB test set (not including the training data). Therefore, the set of stimuli was composed of $50 \times 3=150$ utterances. 
Twelve graduate and undergraduate students participated in the experiment.

In the intelligibility test, evaluation was made using a five-point scale (5: very good, 1 : very bad).

In the test of similarity to dialogue speech, the subjects were instructed to evaluate whether the stimulus sounded like dialogue speech or monologue speech. Prior to the experiment, we first explained the terms "dialogue" and "monologue" and presented 10 natural utterances for each type of speech to the subjects. Then, the subjects practiced evaluating the similarity to dialogue speech for 10 natural utterances composed of 5 examples of dialogue speech and 5 of monologue speech. After that, they evaluated the similarity of the stimuli to dialogue speech on a five-point scale (5: sounds like dialogue speech, 1: sounds like monologue speech).

\subsection{Results}

The results of the intelligibility test are shown in Fig. 1. The mean opinion score was 3.03, 3.96, and 3.54 for the UUDB, CSJ, and ADAPT models, respectively. The result of Tukey's multiple comparison test revealed that the differences of all these combinations were significant $(p<0.05)$. Therefore, the intelligibility of synthesized dialogue speech was improved by the speech mode adaptation $(3.0 \rightarrow 3.5)$. However, a decrease in intelligibility was found before and after the adaptation $(4.0 \rightarrow 3.5)$.

The results of the test of similarity to dialogue speech are shown in Fig. 2. The mean opinion score was 3.74, 2.57, and 3.78 for the UUDB, CSJ, and ADAPT models, respectively. The result of Tukey's multiple comparison test revealed that the difference between UUDB and CSJ, and that between CSJ and ADAPT, were significant $(p<0.05)$. Therefore, the utterances synthesized with the ADAPT model sounded like dialogue speech to a similar extent to the utterances synthesized with the UUDB model. The score for ADAPT was obviously higher than that of the CSJ model, where a 1.2-point improvement was observed.

Therefore, it is considered that the intelligibility can be

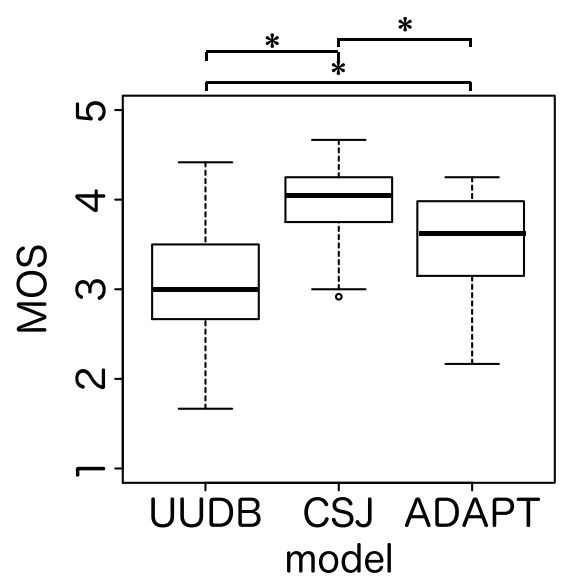

Fig. 1 Mean of opinion scores for the intelligibility test $(*: p<.05)$. improved without sacrificing similarity to dialogue speech by the speech mode adaptation from monologue to dialogue.

\section{Discussion}

The number of leaf nodes of the F0 decision tree of the CSJ model and the UUDB model was 1951 and 352, respectively. This implies that a more fine-grained model was obtained with the CSJ dataset than with the UUDB dataset. Because the ADAPT model had the same complexity as the CSJ model, it is reasonable that the ADAPT model has higher intelligibility than the UUDB model.

An example parameter (log F0) synthesized with the CSJ model and with the ADAPT model are shown in Fig. 3. It can be seen that the phoneme duration changed after the speech mode adaptation (best understood at the end of utterance).

The scatter plot of natural phoneme duration versus that predicted by the CSJ and ADAPT models is shown in Figs. 4 (a) and (b), respectively. The horizontal axis corresponds to the actual duration of the test utterances, and the vertical axis corresponds to the predicted duration for the utterances. Natural speech contains phonemes longer than $200 \mathrm{~ms}$, but the CSJ model estimated the duration for the phonemes to be shorter than the actual lengths. Many of these reflect syllable prolongation, which is a characteristic

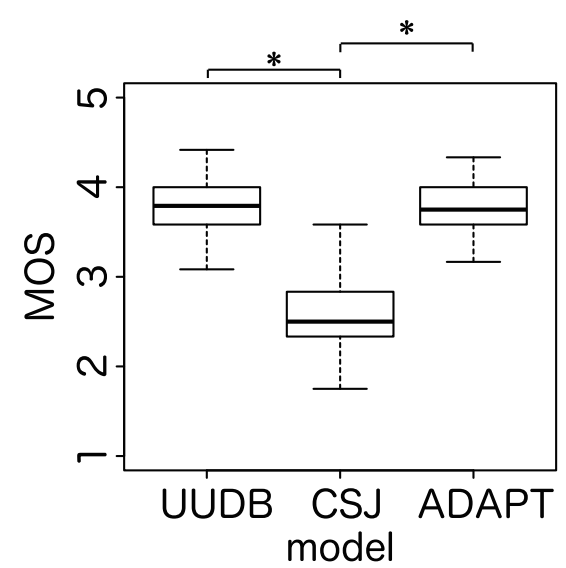

Fig. 2 Mean of opinion scores for the test of likeness to dialogue speech $(*: p<.05)$.

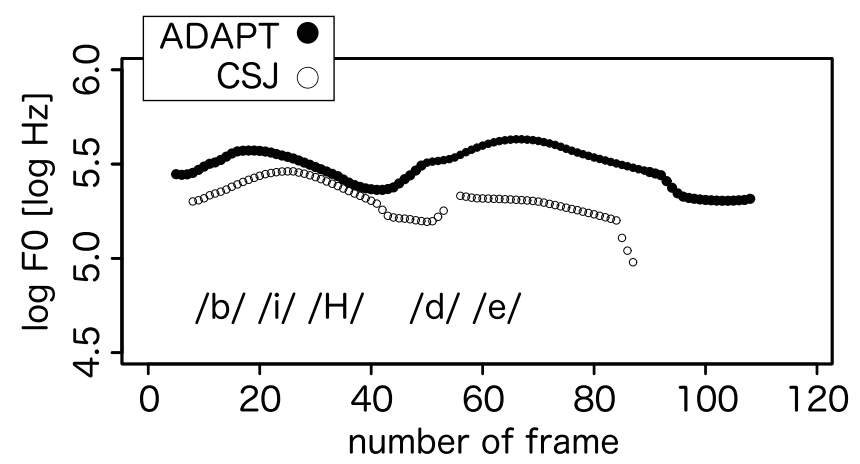

Fig. 3 Examples of F0 contour of the synthesized speech. 


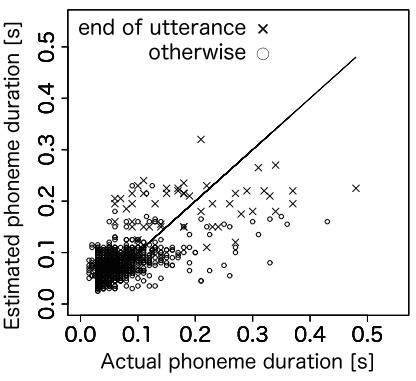

(a):CSJ

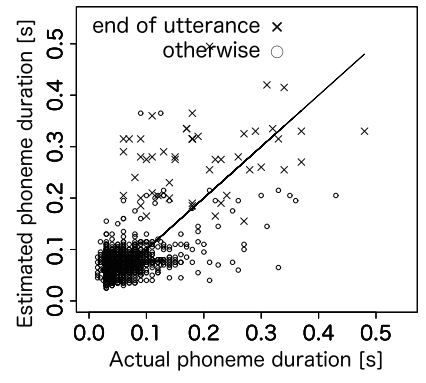

(b):ADAPT
Fig. 4 Scatter plots of phoneme duration.

Table 1 Cepstral distance from the center of the vowel space.

\begin{tabular}{ccc} 
& CSJ $[\mathrm{dB}]$ & ADAPT $[\mathrm{dB}]$ \\
\hline$/ \mathrm{a} /$ & 3.51 & 3.52 \\
$\mathrm{e} /$ & 2.21 & 2.07 \\
$/ \mathrm{i} /$ & 4.24 & 3.35 \\
$\mathrm{/o} /$ & 3.21 & 3.05 \\
$/ \mathrm{u} /$ & 1.37 & 1.62 \\
\hline
\end{tabular}

of dialogue speech that typically appears at phrase boundaries. On the other hand, the duration of some of these phonemes was corrected to the expected length by applying the speech mode adaptation. Therefore, it is likely that the changes to phoneme duration helped make the synthesized speech sound more like dialogue speech.

As described in Sect. 3.2, the intelligility decreased after applying the adaptation. A possible cause of this degradation is duration. The adaptation occasionally brings a drastic change in the state duration, which results in less intelligible speech. Also, the intonation of several stimuli synthesized by ADAPT model sounds unnatural, which may have affected the evaluation.

Table 1 shows the spectral difference calculated from the distance between the mean vector of the synthesized mel-cepstral coefficients of each vowel and its average over all vowels, which has been used to assess the vowel space reduction of spontaneous speech [10]. Shorter distance implies that the vowel is more neutralized, which indicates a more sloppy pronunciation. From Table 1, it is understood that the speech mode adaptation did not affect the vowel space much, except for the vowel /i/ that got $0.89 \mathrm{~dB}$ closer to the center of the vowel space. However, there is still the possibility that systematic effects of spectral features other than the vowel space exist on the perception of speaking style. Further investigation will be needed to understand if such effects exist.

\section{Conclusions}

In this study, we aimed to improve the quality of dia- logue speech synthesis. We proposed a high-quality dialogue speech synthesis based on the speech mode adaptation, where the base model, representing fine-grained prosodic/phonetic classes, is first constructed with a largescale monologue corpus, and then transformed into the target style using broad regression classes constructed with a dialogue corpus. The result of a subjective evaluation of synthesized speech revealed that this method effectively improves the quality of dialogue speech synthesis.

In the future, we will analyze the differences between monologue speech and dialogue speech in more detail, and explore the conversion method focusing on the differences.

\section{References}

[1] K. Tokuda, T. Yoshimura, T. Masuko, T. Kobayashi, and T. Kitamura, "Speech parameter generation algorithms for HMM-based speech synthesis," Proc. ICASSP 2000, pp.1315-1318, 2000.

[2] H. Zen, K. Tokuda, T. Masuko, T. Kobayasih, and T. Kitamura, "A hidden semi-Markov model-based speech synthesis system," IEICE Trans. Inf. \& Syst., vol.E90-D, no.5, pp.825-834, May 2007.

[3] H. Mori and T. Hitomi, "Annotating conversational speech for corpus-based dialogue speech synthesizer - A first step," Proc. Oriental COCOSDA 2012, pp.135-140, 2012.

[4] T. Nagata, H. Mori, and T. Nose, "Dimensional paralinguistic information control based on multiple-regression HSMM for spontaneous dialogue speech synthesis with robust parameter estimation," Speech Communication, vol.88, pp.137-148, 2017.

[5] K. Maekawa, "Corpus of spontaneous Japanese: Its design and evaluation," Proc. ISCA \& IEEE Wkshp. Spontaneous Speech Processing and Recognition (SSPR2003), pp.7-12, 2003.

[6] H. Mori, T. Satake, M. Nakamura, and H. Kasuya, "Constructing a spoken dialogue corpus for studying paralinguistic information in expressive conversation and analyzing its statistical/acoustic characteristics," Speech Communication, vol.53, no.1, pp.36-50, 2011.

[7] H. Kawahara, I. Masuda-Katsuse, and A. de Cheveigné, "Restructuring speech representations using a pitch-adaptive timefrequency smoothing and an instantaneous-frequency-based F0 extraction: Possible role of a reptitive structure in sounds," Speech Communication, vol.27, no.3-4, pp.187-207, 1999.

[8] J. Yamagishi and T. Kobayashi, "Average-voice-based speech synthesis using HSMM-based speaker adaptation and adaptive training," IEICE Trans. Inf. \& Syst., vol.E90-D, no.2, pp.533-543, Feb. 2007.

[9] S. Young, G. Evermann, M. Gales, T. Hain, D. Kershaw, X.Y. Liu, G. Moore, J. Odell, D. Ollason, D. Povey, V. Valtchev, and P. Woodland, The HTK Book (for HTK Version 3.4), Cambridge University Engineering Department, 2006.

[10] M. Nakamura, K. Iwano, and S. Furui, "Differences between acoustic characteristics of spontaneous and read speech and their effects on speech recognition performance," Computer Speech \& Language, vol.22, no.2, pp.171-184, 2008. 